\title{
EVALUACIÓN DE UN PROCESO NOVEDOSO DE BENEFICIADO EN SECO DEL GRANO DE CAÑAHUA (Chenopodium pallidicaule Aellen), BASADO EN LA APLICACIÓN DE UN LECHO FLUIDIZADO TIPO SURTIDOR
}

\author{
Carmen Carla Quiroga Ledezma, Adrián Jaime Ortiz Mérida y Carlos Ramiro Escalera Vásquez
}

\section{RESUMEN}

En este trabajo se evaluó la aplicación de un lecho fluidizado tipo surtidor (LFTS) en el proceso de remoción del perigonio y episperma en el grano de cañahua (Chenopodium pallidicaule Aellen), variedad Illimani y ecotipo lasta rosada, provenientes de la provincia de Pacajes del departamento de La Paz. Para ello, se emplearon dos reactores de vidrio cilíndrico-cónicos de 7,44 y $14,34 \mathrm{~cm}$ de diámetro interno $\left(\mathrm{D}_{\mathrm{c}}\right)$, con diámetros de ingreso de aire de 1 a $5 \mathrm{~mm}$ $\left(\mathrm{d}_{\mathrm{i}}\right)$, conectados a un compresor de $400 \mathrm{~L} / \mathrm{min}$ de capacidad y provistos de un medidor de flujo de $500 \mathrm{~L}$ estándar $/$ min. Muestras de cañahua fueron procesadas de acuerdo con un diseño experimental multifactorial de 32 corridas, evaluándose el efecto de los factores: variedad/ecotipo (Illimani y lasta rosada), $\mathrm{D}_{\mathrm{c}}(7,44 \mathrm{y} 14,34 \mathrm{~cm}), \mathrm{d}_{\mathrm{i}}(1 \mathrm{y} 3 \mathrm{~mm})$ y altura de lecho $\left(\mathrm{H}_{\mathrm{L}}\right)(7,5$ y $12,5 \mathrm{~cm})$, sobre el porcentaje de remoción del perigonio y episperma, el consumo específico de energía y la calidad morfológica y nutricional del grano de cañahua. Los resultados del análisis de varianza multifactorial revelan que todos los factores estudiados son preponderantes en la remoción del perigonio y episperma del grano de cañahua, especialmente el factor $d_{i}$, y en el consumo especifico de energía, principalmente los factores $\mathrm{D}_{\mathrm{c}} \mathrm{y} \mathrm{H}_{\mathrm{L}}$. Las mayores eficacias de remoción de las capas que cubren el grano de cañahua corresponden a las condiciones de operación del LFTS con menores $d_{i}$ y $D_{c}$ y mayores $H_{L}$, aunque estas condiciones demandan precisamente los mayores consumos específicos de energía por unidad de masa de cañahua tratada. Los mayores porcentajes de remoción de masa de la superficie del grano de cañahua se dan en los primeros 5 minutos del proceso, siendo la variedad Illimani la que reporta mayores porcentajes de remoción. En las mejores condiciones de operación, a los 20 - 25 minutos de procesamiento se logra un grano libre de perigonio y episperma, llamado comúnmente "grano perlado", sin daño aparente en la morfología del grano y con una calidad similar a una muestra escarificada y lavada que se comercializa en el mercado nacional e internacional, y con los mismos contenidos iniciales de proteínas y lípidos. El material removido que se colecta es un subproducto con altos contenidos de minerales y fibra dietética, que podría tener aplicaciones industriales, aunque hay que tener presente la existencia de saponinas en el mismo. Como conclusión general se puede afirmar que la aplicación de un LFTS a escala laboratorio es idónea para la remoción del perigonio y episperma del grano de variedades y ecotipos de cañahua y constituye una alternativa a los procesos tradicionales y convencionales que se usan actualmente.

Palabras Clave: Cañahua, Cañihua, Chenopodium pallidicaule Aellen, Lecho Fluidizado, Grano Andino, Pseudocereal.

DOI: $10.23881 /$ idupbo.018.1-2i 